\title{
Comparison of effectiveness and adverse effects of gefitinib, erlotinib and icotinib among patients with non-small cell lung cancer: A network meta-analysis
}

\author{
YUANYUAN LIU ${ }^{1,2 *}$, YU ZHANG ${ }^{3 *}$, GANGLING FENG ${ }^{1,2}$, QIANG NIU ${ }^{1,2}$, SHANGZHI XU $^{1,2}$, \\ YIZHONG YAN $^{1,2}$, SHUGANG LI ${ }^{1,2}$ and MINGXIA JING ${ }^{1,2}$ \\ ${ }^{1}$ Department of Public Health; ${ }^{2}$ Key Laboratory of Xinjiang Endemic and Ethnic Diseases, School of Medicine; ${ }^{3}$ Department \\ of Obstetrics and Gynecology, The First Affiliated Hospital, School of Medicine, \\ Shihezi University, Shihezi, Xinjiang 832002, P.R. China
}

Received November 1, 2016; Accepted April 21, 2017

DOI: $10.3892 /$ etm.2017.5094

\begin{abstract}
The present network meta-analysis aimed to compare the effectiveness and adverse effects of gefitinib, erlotinib and icotinib in the treatment of patients with non-small cell lung cancer (NSCLC). Two reviewers searched the Cochrane, PubMed, Embase, ScienceDirect, China National Knowledge Infrastructure, VIP Database for Chinese Technical Periodicals and Wanfang databases for relevant studies. Studies were then screened and evaluated, and data was extracted. End-points evaluated for NSCLC included complete response (CR), partial response (PR), stable disease (SD), progressive disease (PD), overall response rate (ORR), disease control rate (DCR), progression-free survival (PFS), median survival time (MST) and adverse effects, including rash, diarrhea, nausea and vomiting, fatigue and abnormal liver function. For the analysis of incorporated studies, RevMan, SPSS, R and Stata software were used. A total of 43 studies with 7,168 patients were included in the network meta-analysis. No significant differences were observed in CR, PR, SD, PD, ORR or DCR between gefitinib, erlotinib and icotinib by using network meta analysis. Compared with gefitinib, erlotinib resulted in a higher rate of nausea and vomiting [adjusted odds ratio $(\mathrm{OR})=2.0 ; 95 \%$ credible interval, 1.1-3.7]. However, no significant differences were observed in the rates of rash, diarrhea, fatigue or abnormal liver function using network meta-analysis. Compared with erlotinib, gefitinib resulted in a lower $\mathrm{SD}$ rate $[\mathrm{OR}=0.86 ; 95 \%$
\end{abstract}

Correspondence to: Professor Shugang $\mathrm{Li}$ or Professor Mingxia Jing, Department of Public Health, School of Medicine, Shihezi University, 4 Beier Road, Shihezi, Xinjiang 832002, P.R. China

E-mail: lishugang@ymail.com

E-mail:shzujmx@sina.com

*Contributed equally

Key words: gefitinib, erlotinib, icotinib, non-small cell lung cancer, network meta-analysis confidence interval (CI): 0.75-0.99; $\mathrm{P}=0.04]$, and lower rates of rash $(\mathrm{OR}=0.45 ; 95 \% \mathrm{CI}, 0.36-0.55 ; \mathrm{P}<0.00001)$, diarrhea $(\mathrm{OR}=0.75 ; 95 \% \mathrm{CI}, 0.61-0.92 ; \mathrm{P}=0.005)$, nausea and vomiting $(\mathrm{OR}=0.47 ; 95 \% \mathrm{CI}, 0.27-0.84 ; \mathrm{P}=0.01)$ and fatigue $(\mathrm{OR}=0.43$; 95\% CI, 0.24-0.76; $\mathrm{P}=0.004)$ through meta-analysis of two congruent drugs. However, gefitinib resulted in a higher rate of rash compared with icotinib $(\mathrm{OR}=1.57$; 95\% CI, 1.18-2.09; $\mathrm{P}=0.002)$. Otherwise, no significant differences were observed in CR, PR, PD, ORR, DCR and abnormal liver function between gefitinib, erlotinib and icotinib through meta-analysis of two congruent drugs. The PFS rate for gefitinib, erlotinib and icotinib was 5.48, 5.15 and 5.81 months, respectively. The MST was 13.26, 13.52, 12.58 months for gefitinib, erlotinib and icotinib, respectively. Gefitinib and icotinib resulted in significantly higher PFS rates compared with erlotinib $(\mathrm{P}<0.05)$. Erlotinib resulted in a significantly longer MST compared with gefitinib and icotinib $(\mathrm{P}<0.05)$. In conclusion, gefitinib, erlotinib and icotinib had similar effectiveness for the treatment of patients with advanced NSCLC. However, gefitinib resulted in a lower frequency of fatigue, and nausea and vomiting, compared with the other two drugs. Icotinib resulted in a lower frequency of rash. Erlotinib resulted in a longer MST, but was also associated with a higher frequency of rash, and nausea and vomiting.

\section{Introduction}

Lung cancer is the one of the leading causes of cancer-associated mortality worldwide (1). The majority ( $~ 80-85 \%)$ of patients with lung cancer patients have non-small cell lung cancer (NSCLC) and $70 \%$ of patients with NSCLC are at an advanced stage by the time of diagnosis (1). In China, lung cancer was a common type of cancer $(48.32 / 100,000)$ and cause of cancer-associated mortality $(39.27 / 100,000)$ in 2011 (2). The burden created by elderly Chinese patients with lung cancer is high (3). For patients with NSCLC who cannot undergo surgery due to having an advanced stage of the disease, platinum-based combination chemotherapy has become the primary treatment $(4,5)$. However, in the majority of patients with NSCLC, the disease will eventually progress 
despite combination therapy (4). Therefore, drugs with better efficacies for the treatment of advanced NSCLC are required.

During the last decade, the development of molecular targeted drugs has increased the effectiveness of NSCLC therapy (5). Epidermal growth factor receptor tyrosine kinase inhibitors (EGFR-TKIs), including gefitinib, erlotinib and icotinib, have been demonstrated to be effective for the treatment of advanced NSCLC with few adverse effects, particularly in patients with NSCLC harboring EGFR mutations $(5,6)$. A multi-institutional randomized phase II trial demonstrated that gefitinib had effective clinical antitumor activity and provided symptomatic relief in patients with NSCLC (7). However, the ISEL study reported that treatment with gefitinib did not significantly improve the survival of patients with NSCLC (8). The BR.21 clinical trial reported that erlotinib, another EGFR-TKI, could prolong survival in patients with NSCLC (9). Although the gefitinib and erlotinib have similar molecular and chemical structures, studies have reported different effects of the two drugs on the survival of patients with NSCLC $(8,9)$. Furthermore, other studies have reported that, compared with gefitinib, erlotinib possesses an improved disease control rate and prolongs progression-free survival with increased median survival time, but has more adverse effects, in patients with NSCLC $(1,10,11)$. The WJOG5108L clinical trial reported that gefitinib and erlotinib have similar efficacies (12). Xia et al (13) reported that icotinib exhibited a similar effectiveness and toxicity compared with gefitinib for the treatment of advanced NSCLC, but icotinib exhibited better disease control rate (DCR) and improved rate of diet and sleep period (13). In addition, the ICOGEN clinical trial demonstrated that icotinib and gefitinib achieved similar clinical cure rates in patients with NSCLC (14).

As demonstrated by the findings discussed above, the effectiveness of gefitinib, erlotinib and icotinib for the treatment of patients with advanced NSCLC remains controversial. To the best of our knowledge, no clinical trials comparing the success rate of gefitinib, erlotinib and icotinib have been reported. In the current study, a network meta-analysis was performed to compare the effectiveness and adverse effects of gefitinib, erlotinib and icotinib for the treatment of patients with advanced NSCLC.

\section{Materials and methods}

Search strategy. According to the patient, intervention, control, outcome (PICO) principle (15), the Cochrane (www.cochranelibrary.com), PubMed (www.ncbi.nlm.nih.gov/pmc), Embase (www.embase.com), ScienceDirect (http://www.sciencedirect. com/), China National Knowledge Infrastructure (www. cnki.net), VIP Database for Chinese Technical Periodicals (http://qikan.cqvip.com/) and Wanfang (http://g.wanfangdata. com.cn/) databases were searched using the following key words gefitinib, erlotinib, icotinib and non-small cell lung cancer. Search strategies were as follows: 'gefitinib' AND 'erlotinib' AND 'non-small cell lung cancer OR non-small cell lung carcinoma OR NSCLC'; 'gefitinib' AND ‘icotinib' AND 'non-small cell lung cancer OR non-small cell lung carcinoma OR NSCLC'; 'erlotinib' AND 'icotinib' AND 'non-small cell lung cancer OR non-small cell lung carcinoma OR NSCLC'.
Eligibility criteria. Studies were selected based on primary screening of the identified abstracts or titles, followed by a secondary screening of the full text. According to the PICO principle, the following eligibility criteria were established: i) Research includes a randomized controlled trial, case-control study or cohort study; ii) subjects are patients with advanced NSCLC confirmed by pathological investigation; iii) intervention measures were gefitinib, erlotinib or icotinib; and iv) end-points included complete response (CR), partial response (PR), stable disease (SD), progressive disease (PD), overall response rate (ORR), DCR, progression-free survival (PFS), median survival time (MST) or adverse effects. Exclusion criteria were as follows: i) Patients having tumors other than NSCLC; ii) initial treatment contained drugs that function via the same molecular mechanism as EGFR-TKIs; and iii) study design devoid of control group. Reviews and case reports were also excluded.

Data extraction and quality assessment. Two reviewers independently evaluated the quality of the studies to be included and then extracted the data. The following information was extracted from eligible studies: First author, date of publication, country of affiliations, type of study, number of patients analyzed, interventions, CR, PR, SD, PD, ORR, DCR, PFS, MST and adverse events.

The quality of randomized controlled trials from the Cochrane network was evaluated in terms of the presence or absence of a randomized patient grouping method, concealed assignment, blinding method, incomplete data reporting, selective reporting and other bias (16). Incomplete data reporting (16) was defined as describing the completeness of outcome data for each main outcome. This includes the numbers in each intervention group (compared with total randomized participants), reasons for attrition/exclusions where reported and any re-inclusions in analyses performed by the review authors. Selective reporting (16) was defined as stating how the possibility of selective outcome reporting was examined by the review authors, and what was found. This includes the study protocol is available and all of the study's pre-specified (primary and secondary) outcomes that are of interest in the review have been reported in the pre-specified way; or the study protocol is not available but it is clear that the published reports include all expected outcomes, including those that were pre-specified. All included studies met the following criteria: Randomized, blinding, incomplete data and selective outcome reporting. Allocation concealment and other sources of bias were unclear in all randomized controlled trials.

The Newcastle-Ottawa Scale questionnaire was used to evaluate the quality of case-control and cohort studies (17). The methods of assessment primarily focused on the following three areas: Choice of subjects, comparability and exposure (outcome). The results of the quality assessment are shown in Tables I and II. The included studies were indicated to be of a good quality (18).

Response assessment.Patient response was assessed according to the Response Evaluation Criteria in Solid Tumors (19), which included CR, PR, SD, PD, ORR, DCR, PFS and MST. $\mathrm{CR}$ and $\mathrm{PR}$ were added together to calculate the ORR, while disease control rate was defined by $\mathrm{CR}, \mathrm{PR}$ and SD. Toxicities 


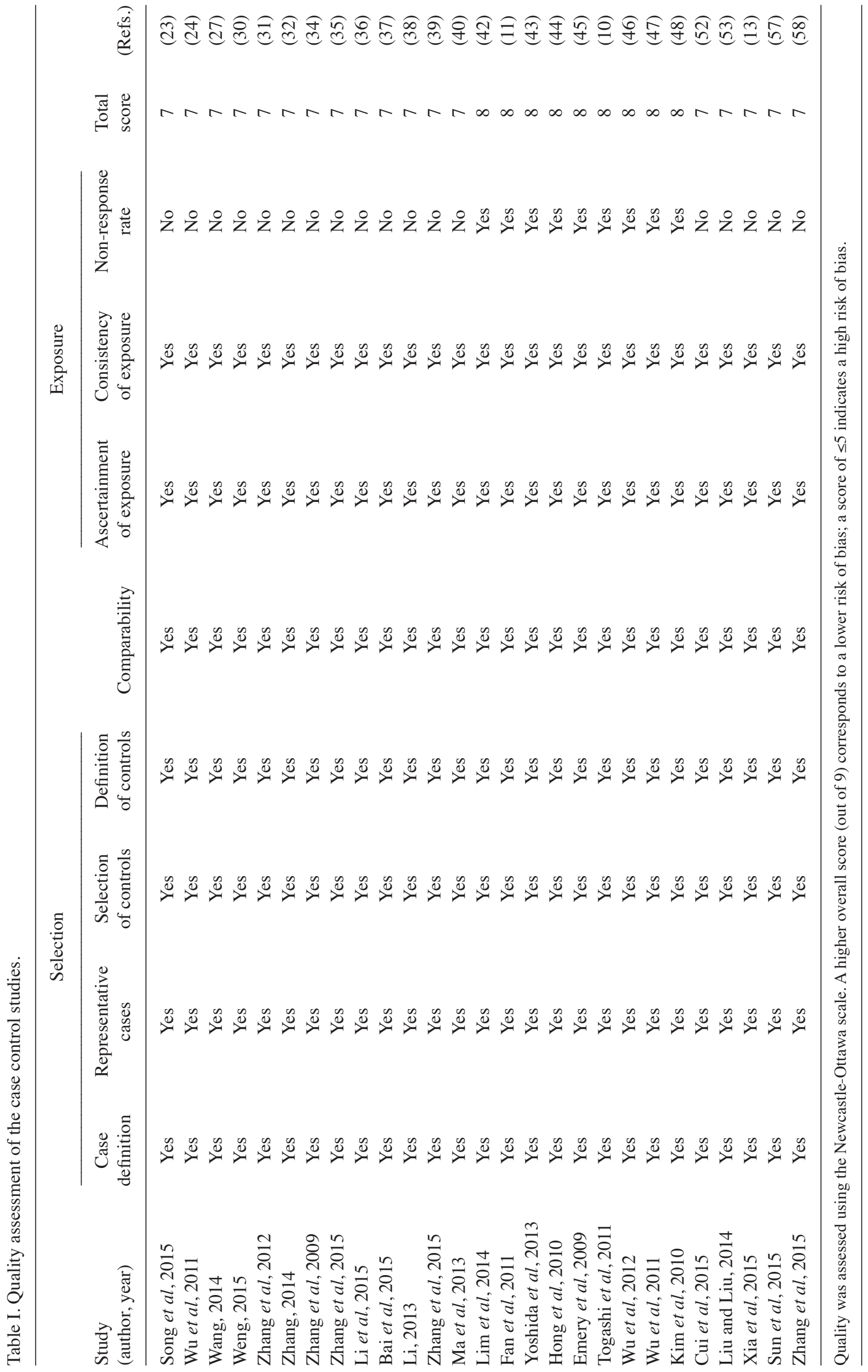




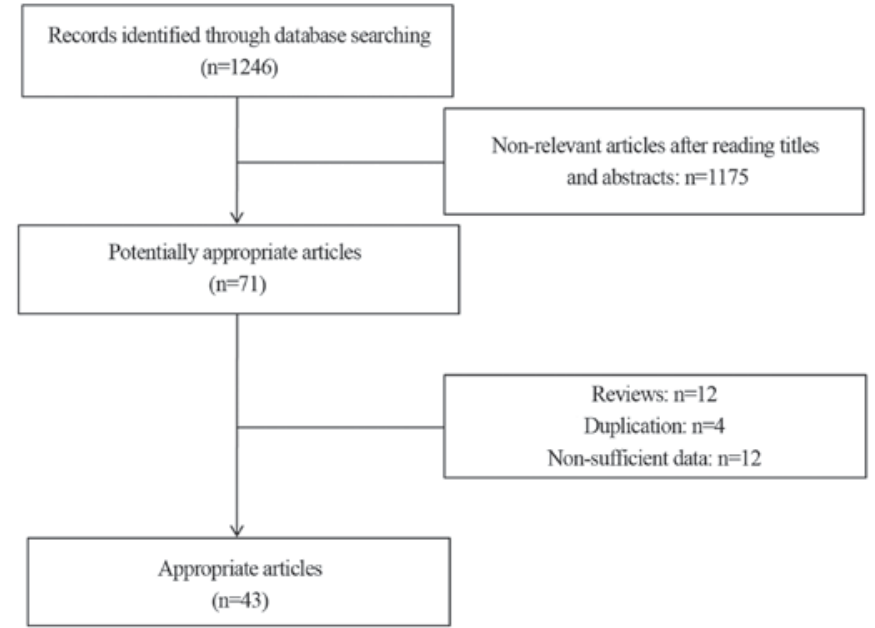

Figure 1. Flow chart illustrating the selection process for articles to be included in the network meta-analysis.

were determined according to the Common Terminology Criteria for Adverse Events version 3.0 (20).

Statistical analysis. Statistical analyses were conducted using RevMan (version 5.2; Cochrane Collaboration, Copenhagen, Denmark), SPSS (version 20.0; IBM Corp., Armonk, NY, USA), R (version 3.3.0; http://mirror.bjtu.edu.cn/cran/) and Stata (version 13.0; StataCorp LLC, College Station, TX, USA) software. R and Stata software were used to perform node-splitting analysis of inconsistencies, network meta-analysis and ranking for drug efficacy. The model for $\mathrm{R}$ software is considered a good indicator when potential scale reduction factor (PSRF) is close to 1 (21). A pooled analysis was performed using RevMan software in order to evaluate indicators $(\mathrm{CR}, \mathrm{PR}, \mathrm{SD}, \mathrm{PD}, \mathrm{ORR}, \mathrm{DCR}$, rash, diarrhea, nausea and vomiting, fatigue and abnormal liver function) among drugs. The adjusted odds ratio (OR) and 95\% confidence interval (CI) or 95\% credible interval (CrI) were used as measures of response for enumeration data. The fixed-effects model (Mantel-Haenszel method) was used for cases with no significant heterogeneity $\left(\mathrm{P}>0.1\right.$ and $\left.\mathrm{I}^{2}<50 \%\right)$. Otherwise, the random-effects model was used. PFS and MST were calculated by the weighted average method using SPSS. The non-parametric Kruskal-Wallis test was used to compare differences in the PFS and MST between groups. Funnel plots were used to assess possible publication bias amongst the included studies. Stata software was used to analyze publication bias with the 'metabias' command and to evaluate sensitivity with the 'metaninf' command. All tests were two-tailed. $\mathrm{P}<0.05$ was considered to indicate a statistically significant difference.

\section{Results}

Literature search. As illustrated in Fig. 1, 1,246 potentially relevant articles were identified through database searching. According to the aforementioned selection criteria, 43 articles $(4,10-14,22-58)$ containing data on 7,168 patients were selected for the network meta-analysis. The characteristics of the eligible studies are described in Table III. 


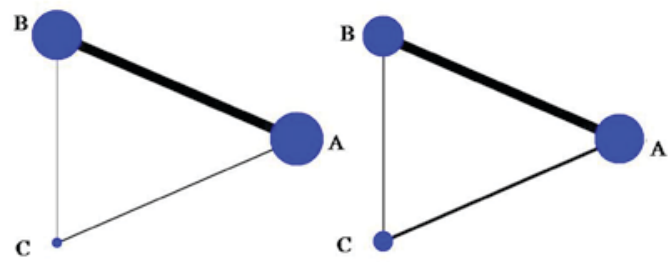

CR

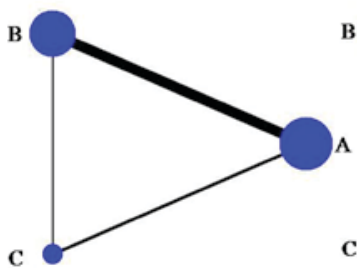

PD



Rash


PR

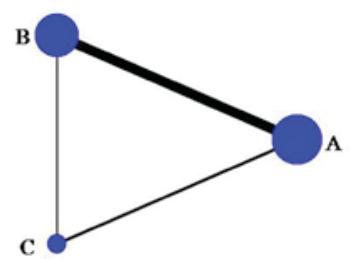

SD



DCR

ORR

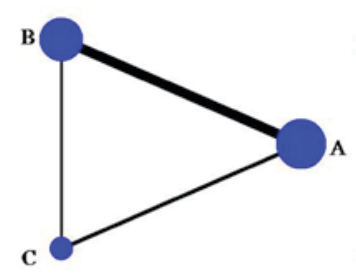

Diarnhea



Nausea vomiting

Figure 2. Network map of the clinical efficacies and adverse events of A, B and C. Node size and line width are based on the number of intervention studies included in the meta-analysis. Larger nodes and thicker lines indicate a higher frequency of intervention with the indicated drug. A, gefitinib; B, erlotinib; C, icotinib; CR, complete response; PR, partial response; SD, stable disease; PD, progressive disease; ORR, overall response rate; DCR, disease control rate.

Network meta-analysis for the clinical effectiveness and adverse effects of gefitinib, erlotinib and icotinib. Network maps (Fig. 2) and network forest plots (Fig. 3) were produced for all indicators. Compared with gefitinib, the network meta-analysis indicated that erlotinib had no significant differences in OR. The ORs for erlotinib were as follows: CR, 1.1 (95\% CrI, 0.65-1.9); PR, 0.97 (95\% CrI, 0.82-1.1); SD, 1.1 (95\% CrI, 0.95-1.3); PD, 0.93 (95\% CrI, 0.77-1.1); ORR, 0.96 (95\% CrI, 0.82-1.1); and DCR, 1.1 (95\% CrI, 0.86-1.3). Icotinib also had no significant differences in OR compared with gefitinib. The ORs for icotinib were as follows: CR, 0.84 (95\% CrI, 0.26-2.4); PR, 1.2 (95\% CrI, 0.89-1.6); SD, 1.1 (95\% CrI, 0.79-1.4); PD, 0.92 (95\% CrI, 0.65-1.3); ORR, 1.2 (95\% CrI, 0.90-1.6); and DCR, 1.3 (95\% CrI, 0.91-1.7).

In terms of adverse events, compared with gefitinib, erlotinib resulted in higher rates of nausea and vomiting $(\mathrm{OR}=2.0 ; 95 \%$ CrI, 1.1-3.7) during network meta-analysis. However, there were no significant differences in OR for rash $(\mathrm{OR}=1.1 ; 95 \% \mathrm{CrI}$, 0.95-1.3), diarrhea (OR=1.3; 95\% CrI, 0.96-1.8), fatigue (OR=2.2; 95\% CrI, 0.84-5.4) or abnormal liver function (OR=1.3; 95\% CrI, 0.85-2.1). Compared with gefitinib, icotinib also had no significant differences in OR for rash $(\mathrm{OR}=1.0 ; 95 \% \mathrm{CrI}, 0.79-1.4)$, diarrhea $(\mathrm{OR}=0.80 ; 95 \% \mathrm{CrI}, 0.50-1.3)$, nausea and vomiting $(\mathrm{OR}=1.2$; 95\% CrI, 0.44-3.8), fatigue $(\mathrm{OR}=3.3$; 95\% CrI, 0.26-39.0) or abnormal liver function $(\mathrm{OR}=0.84 ; 95 \% \mathrm{CrI}, 0.46-1.6)$.
Meta-analysis of two congruent drugs. In the meta-analysis, compared with erlotinib, gefitinib produced lower rates of SD (OR=0.86; 95\% CI, 0.75-0.99; $\mathrm{P}=0.04$; Fig. 4), rash $(\mathrm{OR}=0.45$; 95\% CI, 0.36-0.55; $\mathrm{P}<0.00001$; Fig. 5), diarrhea $(\mathrm{OR}=0.75 ; 95 \%$ CI, 0.61-0.92; $\mathrm{P}=0.005$; Fig. 6$)$, nausea and vomiting $(\mathrm{OR}=0.47$; $95 \%$ CI, 0.0.27-0.84; $\mathrm{P}=0.01$; Fig. 7$)$ and fatigue $(\mathrm{OR}=0.43 ; 95 \%$ CI, 0.24-0.76; $\mathrm{P}=0.004$; Fig. 8). However, gefitinib produced a higher incidence of rash compared with icotinib $(\mathrm{OR}=1.57$; 95\% CI, 1.18-2.09; $\mathrm{P}=0.002$; Fig. 9). No significant differences were observed between gefitinib and icotinib for CR, PR, PD, ORR, DCR, diarrhea or abnormal liver function (Table IV).

Ranking of interventions. Interventions were ranked by how often they caused certain adverse effects (Table V). Erlotinib was observed to produce the highest rate of SD, followed by icotinib. However, erlotinib was also associated with highest risk of rash, followed by gefitinib (data not shown). Icotinib was associated with the highest risk of diarrhea, followed by gefitinib. Erlotinib was associated with the highest risk of nausea and vomiting, followed by icotinib. Icotinib was associated with the highest risk of fatigue, followed by erlotinib.

Analysis of inconsistency and convergence. Using the consistency model, the PSRF for all indicators of clinical efficacies 


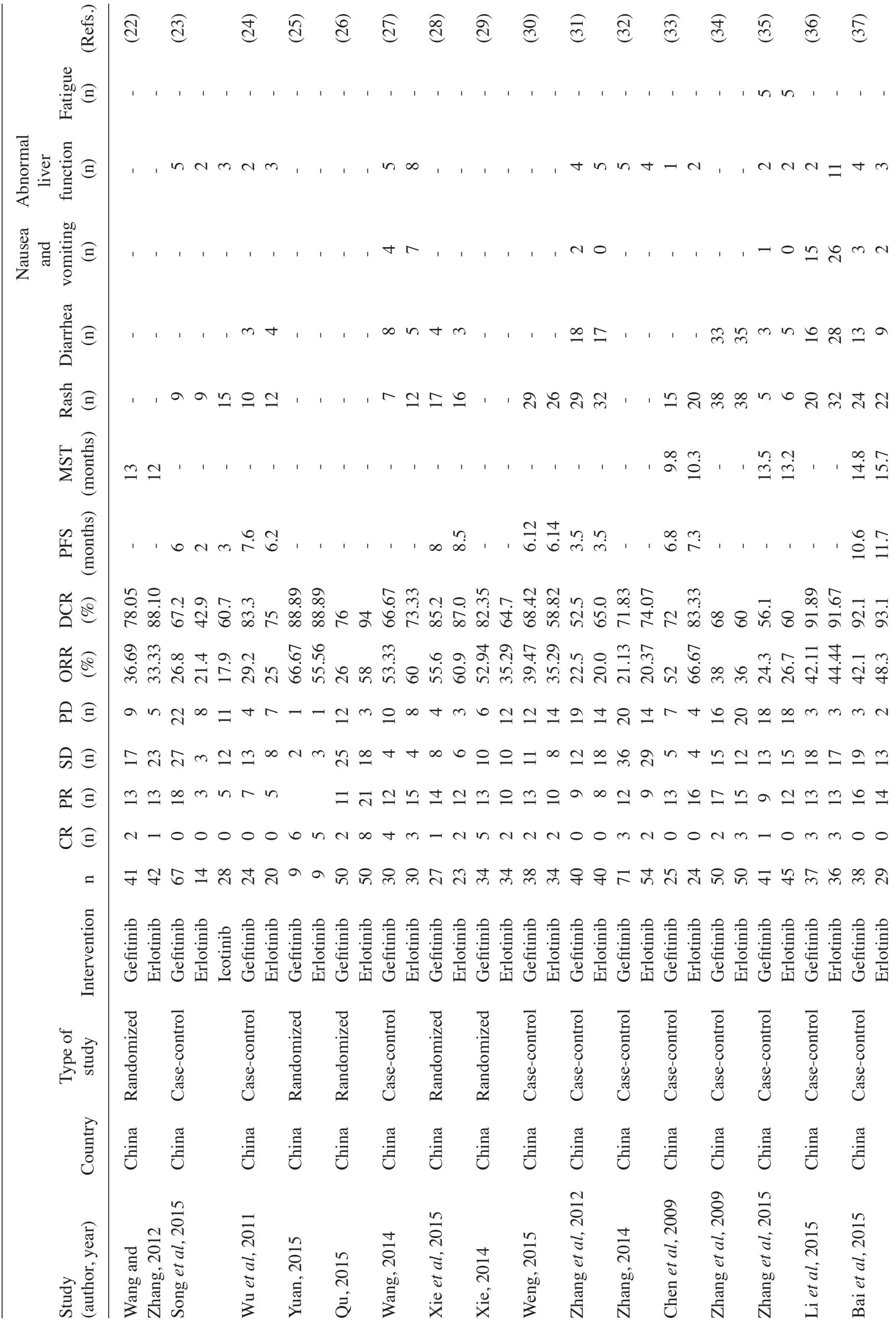




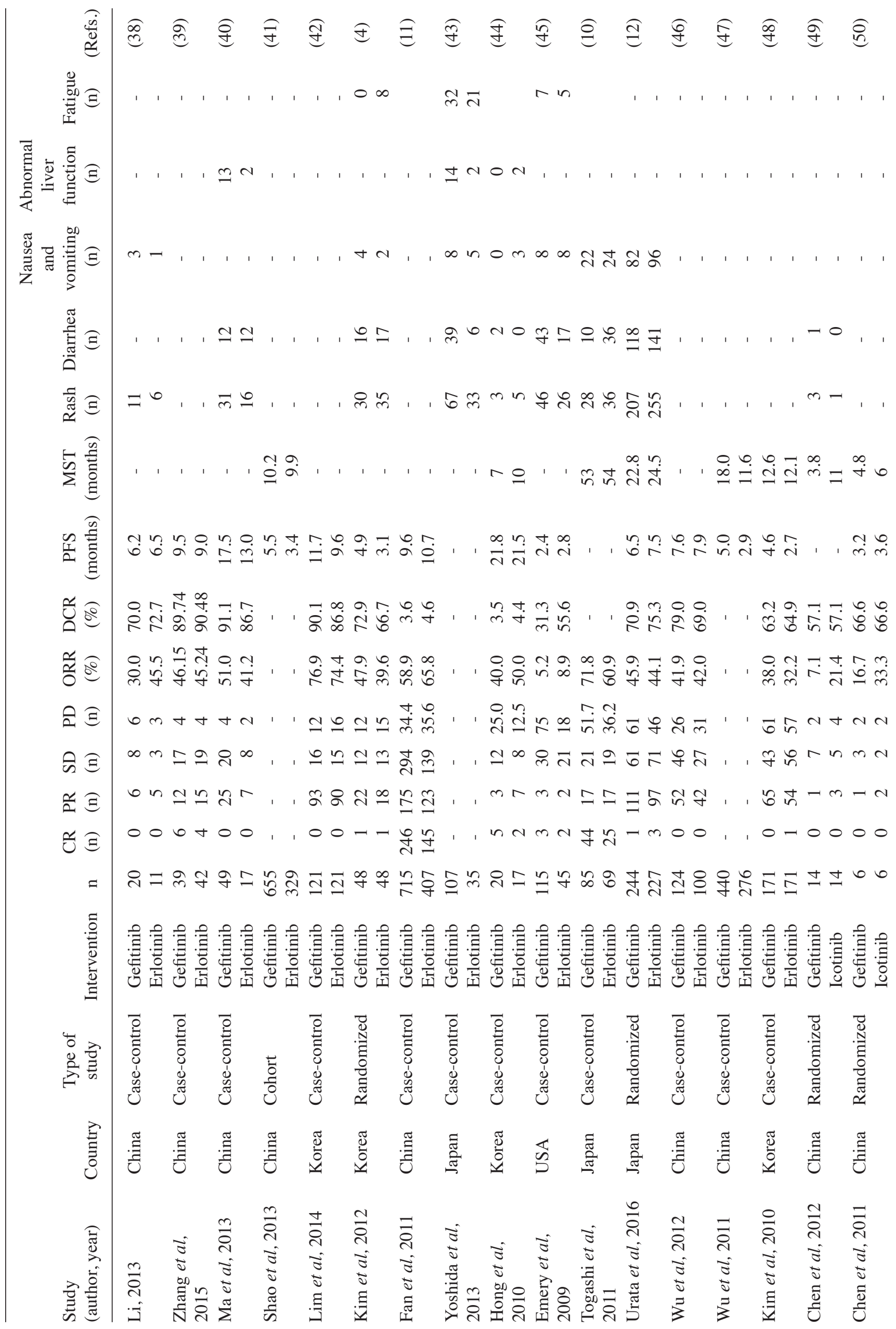









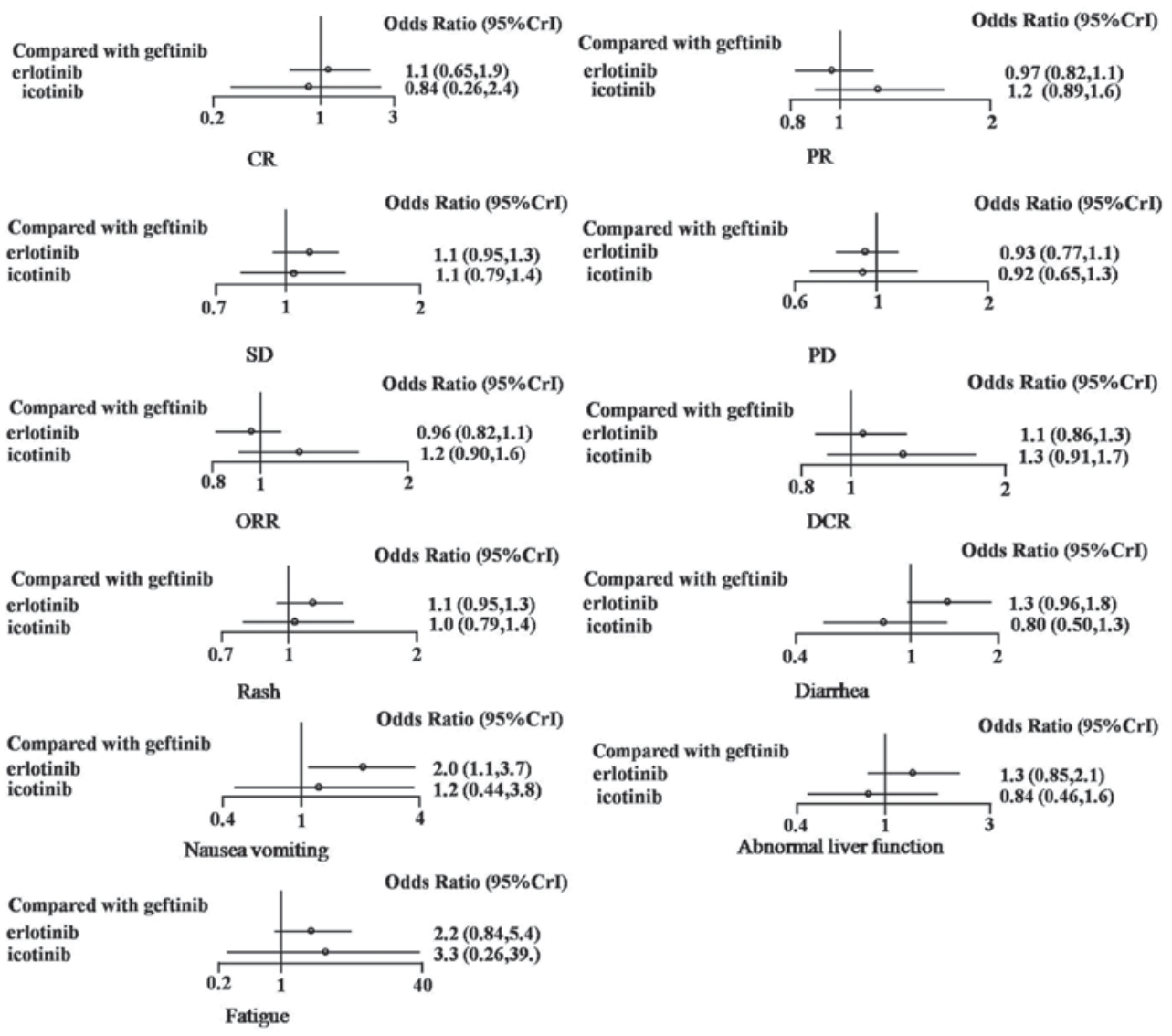

Figure 3. Forest plot of the clinical efficacies and adverse events in the network meta-analysis. CrI, credible intervals; CR, complete response; PR, partial response; SD, stable disease; PD, progressive disease; ORR, overall response rate; DCR, disease control rate.

\begin{tabular}{|c|c|c|c|c|c|c|c|c|c|c|}
\hline Study or Subgroup & \multicolumn{2}{|c|}{ Gefitinib } & \multicolumn{2}{|c|}{ Erlotinib } & Weight & $\begin{array}{c}\text { Odds Ratio } \\
\text { M-H,Fixed,95\%CI }\end{array}$ & \multicolumn{4}{|c|}{$\begin{array}{c}\text { Odds Ratio } \\
\text { M-H,Fixed,95\%CI }\end{array}$} \\
\hline Bai H 2015(37) & 19 & 38 & 13 & 29 & $1.7 \%$ & $1.23[0.47,3.25]$ & & & & \\
\hline Chen XP 2009(33) & 5 & 25 & 4 & 24 & $0.7 \%$ & $1.25[0.29,5.35]$ & & & & \\
\hline Emery IF 2009(45) & 30 & 115 & 21 & 45 & $5.1 \%$ & $0.40[0.20,0.83]$ & & - & & \\
\hline Fan WC 2011(11) & 175 & 715 & 123 & 407 & $26.9 \%$ & $0.75[0.57,0.98]$ & & 을 & & \\
\hline Hong J 2010(44) & 3 & 20 & 7 & 17 & $1.5 \%$ & $0.25[0.05,1.20]$ & & & & \\
\hline Kim ST 2010(48) & 43 & 171 & 56 & 171 & $9.5 \%$ & $0.69[0.43,1.10]$ & & & & \\
\hline Kim ST 2012(4) & 12 & 48 & 13 & 48 & $2.2 \%$ & $0.90[0.36,2.23]$ & & & & \\
\hline Li L 2013(38) & 8 & 20 & 3 & 11 & $0.5 \%$ & $1.78[0.36,8.81]$ & & & & \\
\hline Li YY $2015(36)$ & 18 & 37 & 17 & 36 & $2.0 \%$ & $1.06[0.42,2.65]$ & & & & \\
\hline Lim SH 2014(42) & 16 & 121 & 15 & 121 & $3.0 \%$ & $1.08[0.51,2.29]$ & & & & \\
\hline Liu AM 2014(53) & 2 & 12 & 1 & 10 & $0.2 \%$ & $1.80[0.14,23.37]$ & & & & \\
\hline Ma YX 2013(40) & 20 & 49 & 8 & 17 & $1.6 \%$ & $0.78[0.26,2.35]$ & & & & \\
\hline Qu WF 2015(26) & 25 & 50 & 18 & 50 & $2.0 \%$ & $1.78[0.80,3.96]$ & & & & \\
\hline Song C 2015(23) & 27 & 67 & 3 & 14 & $0.7 \%$ & $2.48[0.63,9.71]$ & & & & \\
\hline Togashi Y 2011(10) & 17 & 85 & 17 & 69 & $3.4 \%$ & $0.76[0.36,1.64]$ & & & & \\
\hline Urata Y 2016(12) & 61 & 244 & 71 & 227 & $12.5 \%$ & $0.73[0.49,1.10]$ & & & & \\
\hline Wang HY 2012(22) & 17 & 41 & 23 & 42 & $3.0 \%$ & $0.59[0.25,1.40]$ & & & & \\
\hline Wang YX 2014(27) & 4 & 30 & 4 & 30 & $0.8 \%$ & $1.00[0.23,4.43]$ & & & & \\
\hline Weng XL 2015(30) & 11 & 38 & 8 & 34 & $1.4 \%$ & $1.32[0.46,3.81]$ & & & & \\
\hline Wu WS 2012(46) & 46 & 124 & 27 & 100 & $4.3 \%$ & $1.59[0.90,2.83]$ & & & & \\
\hline Wu X 2011(24) & 13 & 24 & 8 & 20 & $0.9 \%$ & $1.77[0.53,5.90]$ & & & & \\
\hline Xie YK 2014(29) & 10 & 34 & 10 & 34 & $1.6 \%$ & $1.00[0.35,2.84]$ & & & & \\
\hline Xie YL 2015(28) & 8 & 27 & 6 & 23 & $1.0 \%$ & $1.19[0.34,4.14]$ & & & & \\
\hline Yuan HF 2015(25) & 2 & 9 & 3 & 9 & $0.5 \%$ & $0.57[0.07,4.64]$ & & & & \\
\hline Zhang CW 2014(32) & 36 & 71 & 29 & 54 & $3.7 \%$ & $0.89[0.44,1.80]$ & & & & \\
\hline Zhang J 2012(31) & 12 & 40 & 18 & 40 & $2.9 \%$ & $0.52[0.21,1.31]$ & & & & \\
\hline Zhang JX 2015(39) & 17 & 39 & 19 & 42 & $2.3 \%$ & $0.94[0.39,2.25]$ & & & & \\
\hline Zhang XQ 2009(34) & 15 & 50 & 12 & 50 & $1.9 \%$ & $1.36[0.56,3.30]$ & & & & \\
\hline Zhang YJ 2015(35) & 13 & 41 & 15 & 45 & $2.2 \%$ & $0.93[0.38,2.29]$ & & & & \\
\hline Total $(95 \% \mathrm{CI})$ & & 2385 & & 1819 & $100.0 \%$ & $0.86[0.75,0.99]$ & & $\theta$ & & \\
\hline Total events & 685 & & 572 & & & & & & & \\
\hline $\begin{array}{l}\text { Heterogeneity: } \mathrm{Chi}^{2}= \\
\text { Test for overall effect }\end{array}$ & $\begin{array}{l}27.11, \mathrm{df}= \\
\mathrm{Z}=2.08\end{array}$ & $\begin{array}{l}=28(\mathrm{P} \\
(\mathrm{P}=0 .\end{array}$ & $\begin{array}{l}=0.51) ; \\
04)\end{array}$ & $I^{2}=0 \%$ & & & 0.01 & 0. & & 100 \\
\hline
\end{tabular}

Figure 4. Forest plot comparing the rate of stable disease following gefitinib and erlotinib treatment for patients with advanced non-small cell lung cancer. M-H, Mantel-Haenszel; CI, confidence interval. 




Figure 5. Forest plot comparing the incidence of rash following gefitinib and erlotinib treatment for patients with advanced non-small cell lung cancer. M-H, Mantel-Haenszel; CI, confidence interval.

\begin{tabular}{|c|c|c|c|c|c|c|c|c|c|}
\hline Study or Subgroup & \multicolumn{2}{|c|}{ Gefitinib } & \multicolumn{2}{|c|}{ Erlotinib } & Weight & \multirow{2}{*}{$\begin{array}{c}\begin{array}{c}\text { Odds Ratio } \\
\text { M-H,Fixed,95\%CI }\end{array} \\
1.16[0.41,3.25]\end{array}$} & \multicolumn{3}{|c|}{$\begin{array}{c}\text { Odds Ratio } \\
\text { M-H,Fixed,95\%CI }\end{array}$} \\
\hline Bai H 2015(37) & 13 & 38 & 9 & 29 & $3.1 \%$ & & & & \\
\hline Emery IF 2009(45) & 43 & 115 & 17 & 45 & $7.0 \%$ & $0.98[0.48,2.00]$ & & & \\
\hline Hong J 2010(44) & 3 & 20 & 5 & 17 & $2.1 \%$ & $0.42[0.08,2.12]$ & & & \\
\hline Kim ST 2012(4) & 16 & 48 & 17 & 48 & $5.2 \%$ & $0.91[0.39,2.12]$ & & & \\
\hline Li YY 2015(36) & 16 & 37 & 28 & 36 & $7.3 \%$ & $0.22[0.08,0.60]$ & & & \\
\hline Liu AM 2014(53) & 5 & 12 & 4 & 10 & $1.2 \%$ & $1.07[0.19,5.91]$ & & & \\
\hline Ma YX 2013(40) & 12 & 49 & 12 & 17 & $6.1 \%$ & $0.14[0.04,0.46]$ & & & \\
\hline Togashi Y 2011(10) & 28 & 85 & 36 & 69 & $12.1 \%$ & $0.45[0.23,0.87]$ & - & & \\
\hline Urata Y 2016(12) & 118 & 279 & 141 & 280 & $37.0 \%$ & $0.72[0.52,1.01]$ & 블 & & \\
\hline Wang YX 2014(27) & 8 & 30 & 5 & 30 & $1.7 \%$ & $1.82[0.52,6.38]$ & & & \\
\hline Wu X 2011(24) & 3 & 24 & 4 & 20 & $1.7 \%$ & $0.57[0.11,2.92]$ & & & \\
\hline Xie YL 2015(28) & 4 & 27 & 3 & 23 & $1.3 \%$ & $1.16[0.23,5.81]$ & & & \\
\hline Yoshida T 2013(43) & 39 & 107 & 6 & 35 & $2.6 \%$ & $2.77[1.06,7.26]$ & & & \\
\hline Zhang J 2012(31) & 18 & 40 & 17 & 40 & $4.3 \%$ & $1.11[0.46,2.68]$ & & & \\
\hline Zhang XQ 2009(34) & 33 & 50 & 35 & 50 & $5.4 \%$ & $0.83[0.36,1.93]$ & & & \\
\hline Zhang YJ 2015(35) & 3 & 41 & 5 & 45 & $2.0 \%$ & $0.63[0.14,2.83]$ & & & \\
\hline Total $(95 \% \mathrm{CI})$ & & 1002 & & 794 & $100.0 \%$ & $0.75[0.61,0.92]$ & & & \\
\hline Total events & 362 & & 344 & & & & & & \\
\hline $\begin{array}{l}\text { Heterogeneity: } \mathrm{Chi}^{2}= \\
\text { Test for overall effect: }\end{array}$ & $\begin{array}{l}27.81, \mathrm{df}= \\
Z=2.80\end{array}$ & $\begin{array}{l}=15(\mathrm{P} \\
(\mathrm{P}=0 .\end{array}$ & $\begin{array}{l}=0.02) ; \\
005)\end{array}$ & $I^{2}=46$ & & & $\begin{array}{ll} \\
0.01 & 0.1 \\
\text { gefitinib }\end{array}$ & erlotinib & 100 \\
\hline
\end{tabular}

Figure 6. Forest plot comparing the incidence of diarrhea following gefitinib and erlotinib treatment for patients with advanced non-small cell lung cancer. M-H, Mantel-Haenszel; CI, confidence interval.

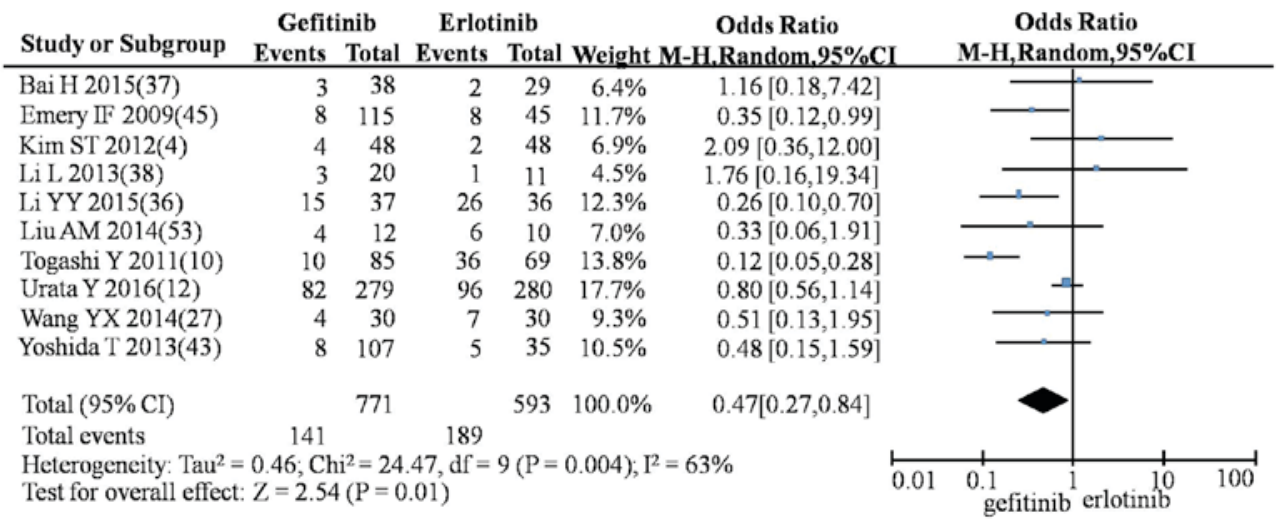

Figure 7. Forest plot comparing the incidence of nausea and vomiting diarrhea following gefitinib and erlotinib treatment for patients with advanced non-small cell lung cancer. M-H, Mantel-Haenszel; CI, confidence interval. 
Table IV. Meta-analysis of the clinical efficacies and adverse events associated with G, E and I.

\begin{tabular}{|c|c|c|c|c|c|c|c|c|c|}
\hline \multirow[b]{2}{*}{ Indicator } & \multirow[b]{2}{*}{ Intervention } & \multirow[b]{2}{*}{$\mathrm{n}$} & \multirow[b]{2}{*}{ OR $(95 \% \mathrm{CI})$} & \multicolumn{3}{|c|}{ Heterogeneity testing } & \multirow[b]{2}{*}{ Model } & \multirow[b]{2}{*}{ Z-value } & \multirow[b]{2}{*}{ P-value } \\
\hline & & & & $\chi^{2}$ & P-value & $\mathrm{I}^{2}$ & & & \\
\hline \multirow[t]{3}{*}{$\mathrm{CR}$} & G vs. E & 14 & $0.90(0.56-1.44)$ & 8.11 & 0.84 & 0 & Fixed & 0.43 & 0.67 \\
\hline & G vs. I & 2 & $1.17(0.53-2.58)$ & 0.34 & 0.56 & 0 & Fixed & 0.40 & 0.69 \\
\hline & E vs. I & 1 & $1.75(0.13-23.70)$ & - & - & - & - & 0.42 & 0.67 \\
\hline \multirow[t]{3}{*}{ PR } & G vs. E & 25 & $1.03(0.88-1.21)$ & 12.57 & 0.97 & 0 & Fixed & 0.41 & 0.68 \\
\hline & G vs. I & 10 & $0.90(0.68-1.19)$ & 4.86 & 0.85 & 0 & Fixed & 0.72 & 0.47 \\
\hline & E vs. I & 4 & $0.63(0.30-1.35)$ & 1.62 & 0.65 & 0 & Fixed & 1.19 & 0.23 \\
\hline \multirow[t]{3}{*}{ SD } & G vs. E & 29 & $0.86(0.75-0.99)$ & 27.11 & 0.51 & 0 & Fixed & 2.08 & 0.04 \\
\hline & G vs. I & 10 & $0.95(0.74-1.23)$ & 4.85 & 0.85 & 0 & Fixed & 0.37 & 0.71 \\
\hline & E vs. I & 5 & $0.94(0.55-1.62)$ & 2.74 & 0.60 & 0 & Fixed & 0.21 & 0.83 \\
\hline \multirow[t]{3}{*}{ PD } & G vs. E & 29 & $1.14(0.99-1.31)$ & 35.44 & 0.16 & 21 & Fixed & 1.87 & 0.06 \\
\hline & G vs. I & 10 & $0.99(0.73-1.34)$ & 5.07 & 0.83 & 0 & Fixed & 0.06 & 0.95 \\
\hline & E vs. I & 5 & $1.48(0.85-2.59)$ & 1.51 & 0.82 & 0 & Fixed & 1.38 & 0.17 \\
\hline \multirow[t]{3}{*}{ ORR } & G vs. E & 29 & $1.03(0.91-1.18)$ & 23.76 & 0.69 & 0 & Fixed & 0.51 & 0.61 \\
\hline & G vs. I & 10 & $0.91(0.69-1.20)$ & 4.81 & 0.85 & 0 & Fixed & 0.68 & 0.49 \\
\hline & E vs. I & 5 & $0.68(0.37-1.25)$ & 1.53 & 0.82 & 0 & Fixed & 1.24 & 0.21 \\
\hline \multirow[t]{3}{*}{ DCR } & G vs. E & 29 & $0.90(0.78-1.03)$ & 37.53 & 0.11 & 25 & Fixed & 1.56 & 0.12 \\
\hline & G vs. I & 10 & $0.85(0.64-1.13)$ & 5.34 & 0.80 & 0 & Fixed & 1.11 & 0.27 \\
\hline & E vs. I & 5 & $0.67(0.39-1.18)$ & 1.51 & 0.82 & 0 & Fixed & 1.38 & 0.17 \\
\hline \multirow[t]{3}{*}{ Rash } & G vs. E & 20 & $0.45(0.36-0.55)$ & 28.08 & 0.08 & 32 & Fixed & 7.30 & $<0.01$ \\
\hline & G vs. I & 9 & $1.57(1.18-2.09)$ & 5.39 & 0.72 & 0 & Fixed & 3.07 & $<0.01$ \\
\hline & E vs. I & 5 & $1.37(0.81-2.30)$ & 3.89 & 0.42 & 0 & Fixed & 1.17 & 0.24 \\
\hline \multirow[t]{3}{*}{ Diarrhea } & G vs. E & 16 & $0.75(0.61-0.92)$ & 27.81 & 0.02 & 46 & Fixed & 2.80 & $<0.01$ \\
\hline & G vs. I & 7 & $1.32(0.94-1.85)$ & 4.20 & 0.65 & 0 & Fixed & 1.60 & 0.11 \\
\hline & E vs. I & 4 & $1.45(0.57-3.72)$ & 1.43 & 0.70 & 0 & Fixed & 0.78 & 0.44 \\
\hline \multirow{3}{*}{$\begin{array}{l}\text { Nausea and } \\
\text { vomiting }\end{array}$} & G vs. E & 10 & $0.47(0.27-0.84)$ & 24.47 & 0.00 & 63 & Random & 2.54 & 0.01 \\
\hline & G vs. I & 3 & $0.99(0.53-1.88)$ & 2.01 & 0.37 & 1 & Fixed & 0.02 & 0.98 \\
\hline & E vs. I & 2 & $0.93(0.19-4.57)$ & 0.00 & 0.95 & 0 & Fixed & 0.09 & 0.93 \\
\hline \multirow{3}{*}{$\begin{array}{l}\text { Abnormal liver } \\
\text { function }\end{array}$} & G vs. E & 13 & $0.73(0.51-1.05)$ & 10.94 & 0.53 & 0 & Fixed & 1.70 & 0.09 \\
\hline & G vs. I & 6 & $1.27(0.77-2.10)$ & 4.33 & 0.50 & 0 & Fixed & 0.94 & 0.35 \\
\hline & E vs. I & 3 & $1.34(0.45-4.00)$ & 0.06 & 0.97 & 0 & Fixed & 0.53 & 0.60 \\
\hline \multirow[t]{3}{*}{ Fatigue } & G vs. E & 4 & $0.43(0.24-0.76)$ & 3.14 & 0.37 & 5 & Fixed & 2.89 & $<0.01$ \\
\hline & G vs. I & 1 & $0.27(0.02-3.67)$ & - & - & - & - & 0.98 & 0.33 \\
\hline & E vs. I & 1 & $0.75(0.08-6.96)$ & - & - & - & - & 0.25 & 0.80 \\
\hline
\end{tabular}

G, gefitinib; E, erlotinib; I, icotinib; n, number of included studies; CR, complete response; PR, partial response; SD, stable disease; PD, progressive disease; ORR, overall response rate; DCR, disease control rate; OR, adjusted odds ratio; CI, confidence interval.

and adverse events was close to 1 (data not shown). Following node-splitting analysis of inconsistencies, no significant differences were observed for CR, PR, SD, PD, ORR, DCR, diarrhea, nausea and vomiting, fatigue or abnormal liver function (data not shown).

PFS and MST. The PFS rate for gefitinib, erlotinib and icotinib was 5.48, 5.15 and 5.81 months, respectively (Table VI). The MST was 13.26, 13.52 and 12.58 months for gefitinib, erlotinib and icotinib, respectively (Table VI). Gefitinib and icotinib had a significantly higher PFS rate compared with erlotinib $(\mathrm{P}<0.01)$; however, no significant difference in PFS was observed between gefitinib and icotinib (Table VI). Erlotinib had a significantly longer MST compared with gefitinib and icotinib $(\mathrm{P}<0.05)$, and gefitinib had a significantly longer MST compared with icotinib $(\mathrm{P}<0.05)($ Table $\mathrm{VI})$.

Publication bias. Funnel plots (Fig. 10) revealed that all included studies were symmetrical in terms of standard error of the effect size and the effect size centered at the comparison-specific pooled effect for CR, PR, SD, PD, ORR and DCR. This indicates that there was minimal publication bias.

Sensitivity analysis. According to sensitivity analysis, there was little difference for the pooled effect among each study for the indexes ORR and DCR (data not shown). 




Figure 8. Forest plot comparing the incidence of fatigue diarrhea following gefitinib and erlotinib treatment for patients with advanced non-small cell lung cancer. M-H, Mantel-Haenszel; CI, confidence interval.

Table V. Ranking of interventions.

A, Stable disease

\begin{tabular}{lccc}
\hline Ranking & Gefitinib (\%) & Erlotinib (\%) & Icotinib (\%) \\
\hline 1 & 2.4 & 71.2 & 26.5 \\
2 & 34.7 & 25.6 & 39.7 \\
3 & 62.9 & 3.2 & 33.8 \\
\hline
\end{tabular}

B, Diarrhea

\begin{tabular}{lccc}
\hline Ranking & Gefitinib $(\%)$ & Erlotinib (\%) & Icotinib (\%) \\
\hline 1 & 1.7 & 95.0 & 3.2 \\
2 & 77.5 & 4.6 & 18.0 \\
3 & 20.8 & 0.4 & 78.8 \\
\hline
\end{tabular}

C, Nausea and vomiting

\begin{tabular}{lccc}
\hline Ranking & Gefitinib (\%) & Erlotinib (\%) & Icotinib (\%) \\
\hline 1 & 65.8 & 0.2 & 34.0 \\
2 & 34.0 & 15.7 & 50.2 \\
3 & 0.1 & 84.1 & 15.8 \\
\hline
\end{tabular}

D, Fatigue

\begin{tabular}{lccc}
\hline Ranking & Gefitinib (\%) & Erlotinib (\%) & Icotinib (\%) \\
\hline 1 & 83.6 & 1.4 & 15.0 \\
2 & 15.8 & 62.0 & 22.2 \\
3 & 0.6 & 36.6 & 62.8 \\
\hline
\end{tabular}

\section{Discussion}

Lung cancer has the highest morbidity and mortality rate of any malignant tumor type, and is most commonly NSCLC $(1,2)$. In total $>50 \%$ of patients diagnosed with NSCLC are at an advanced stage of the disease (40). Platinum-based combination chemotherapy is the most common course of treatment for patients with NSCLC, but its clinical efficacy is limited (4).
Table VI. Comparison of PFS and MST.

\begin{tabular}{lcc}
\hline Intervention & PFS (months) & MST (months) \\
\hline Gefitinib & $5.48^{\mathrm{a}}$ & $13.26^{\mathrm{a}, \mathrm{b}}$ \\
Erlotinib & 5.15 & $13.52^{\mathrm{a}, \mathrm{c}}$ \\
Icotinib & $5.81^{\mathrm{c}}$ & $12.58^{\mathrm{b}, \mathrm{c}}$ \\
\hline
\end{tabular}

${ }^{\mathrm{a}} \mathrm{P}<0.05$ gefitinib vs. erlotinib; ${ }^{\mathrm{b}} \mathrm{P}<0.05$ gefitinib vs. icotinib; ${ }^{\mathrm{C}} \mathrm{P}<0.05$ erlotinib vs. icotinib.

Targeted therapy, including gefitinib, erlotinib and icotinib, has improved the treatment of advanced NSCLC $(7,9,59)$. However, there remains controversy surrounding the effectiveness and adverse effects of these three drugs $(1,10-14,60)$.

In the current meta-analysis, no significant differences for CR, PR, SD, PD, ORR, DCR, rash, diarrhea, fatigue or abnormal liver function were observed between gefitinib, erlotinib and icotinib by using network meta analysis. The frequency of fatigue, and nausea and vomiting, was lower for gefitinib compared with icotinib or erlotinib. In addition, the MST was longer for erlotinib compared with the other two drugs. Furthermore, the frequency of rash for icotinib was lower compared with the other two drugs. The present study revealed that the three drugs had similar efficacies for the treatment of patients with advanced NSCLC. Overall, gefitinib had a lower frequency of nausea and vomiting and fatigue, while erlotinib has a longer MST, but a higher frequency for rash and nausea and vomiting. A lower frequency of rash may occur with icotinib.

The results of the network meta-analysis conducted in the present study revealed that there were no significant differences between the efficacies of gefitinib, erlotinib or icotinib for the treatment of NSCLC. All three targeted drugs act as EGFR-TKIs and bind to the Mg-ATP binding site of the EGFR tyrosine kinase catalytic domain competitively $(45,58)$. This inhibits EGFR phosphorylation and subsequent signal transduction. Thus, these drugs have antitumor activity $(10,59,61)$. Since these drugs function via the same molecular mechanism, they may have similar efficacies in the treatment of patients with NSCLC. In the current study, no significant statistical differences were observed in CR, PR, PD, ORR or DCR by performing meta-analysis of two congruent drugs. 


\begin{tabular}{|c|c|c|c|c|c|c|c|c|c|}
\hline Study or Subgroup & \multicolumn{2}{|c|}{ Gefitinib } & \multicolumn{2}{|c|}{ Icotinib } & Weight & $\begin{array}{c}\text { Odds Ratio } \\
\text { M-H,Fixed,95\%CI }\end{array}$ & & \multicolumn{2}{|c|}{$\begin{array}{c}\text { Odds Ratio } \\
\text { M-H,Fixed,95\%CI }\end{array}$} \\
\hline Chen JH 2012(49) & 3 & 14 & 1 & 14 & $1.0 \%$ & $3.55[0.32,39.14]$ & & & \\
\hline Cui HQ 2015(52) & 13 & 21 & 31 & 49 & $9.4 \%$ & $0.94[0.33,2.71]$ & & & - \\
\hline Cui HZ 2015(51) & 8 & 28 & 3 & 28 & $2.9 \%$ & $3.33[0.78,14.23]$ & & & \\
\hline Lin WX 2014(54) & 11 & 24 & 10 & 24 & $7.2 \%$ & $1.18[0.38,3.71]$ & & & \\
\hline Liu AM 2014(53) & 4 & 12 & 2 & 8 & $2.1 \%$ & $1.50[0.20,11.09]$ & & & \\
\hline Shi Y $2013(14)$ & 98 & 199 & 81 & 200 & $54.6 \%$ & $1.43[0.96,2.12]$ & & & -1 \\
\hline Song C 2015(23) & 53 & 67 & 15 & 28 & $5.9 \%$ & $3.28[1.27,8.47]$ & & & $\rightarrow$ \\
\hline Xia J 2015(13) & 85 & 93 & 112 & 126 & $10.9 \%$ & $1.33[0.53,3.31]$ & & & $f=$ \\
\hline Xu LJ 2015(55) & 10 & 40 & 6 & 40 & $6.0 \%$ & $1.89[0.61,5.82]$ & & & \\
\hline Total $(95 \% \mathrm{CI})$ & & 498 & & 517 & $100.0 \%$ & $1.57[1.18,2.09]$ & & & $\bullet$ \\
\hline Total events & 285 & & 261 & & & & & & \\
\hline $\begin{array}{l}\text { Heterogeneity: } \mathrm{Chi}^{2}= \\
\text { Test for overall effect }\end{array}$ & $\begin{array}{l}5.39, \mathrm{df} \\
\mathrm{Z}=3.07\end{array}$ & $\begin{array}{l}=8(\mathrm{P}= \\
(\mathrm{P}=0\end{array}$ & $=0.72) ; I^{2}$ & $=0 \%$ & & & 0.01 & $\underset{\text { gefitinib }}{0.1}$ & ${ }^{1}$ icotinib $^{1}$ \\
\hline
\end{tabular}

Figure 9. Forest plot comparing the incidence of rash diarrhea following gefitinib and erlotinib treatment for patients with advanced non-small cell lung cancer. M-H, Mantel-Haenszel; CI, confidence interval.
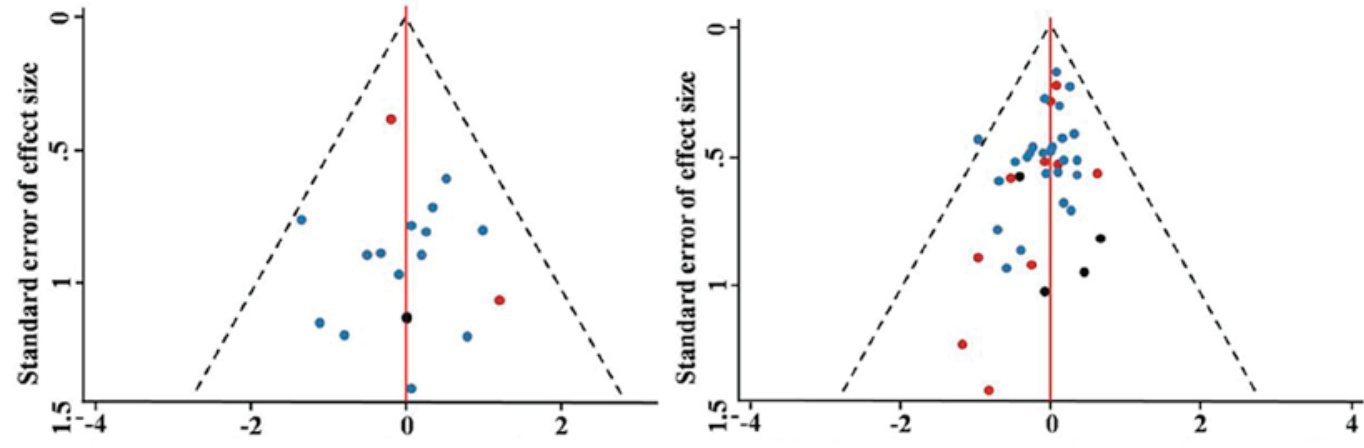

Effect size centered at comparison-specific pooled effect Effect size centered at comparison-specific pooled effect $(y x X-\mu x Y)$

$($ (yixY- $\mu x y)$

$\mathrm{CR}$

PR
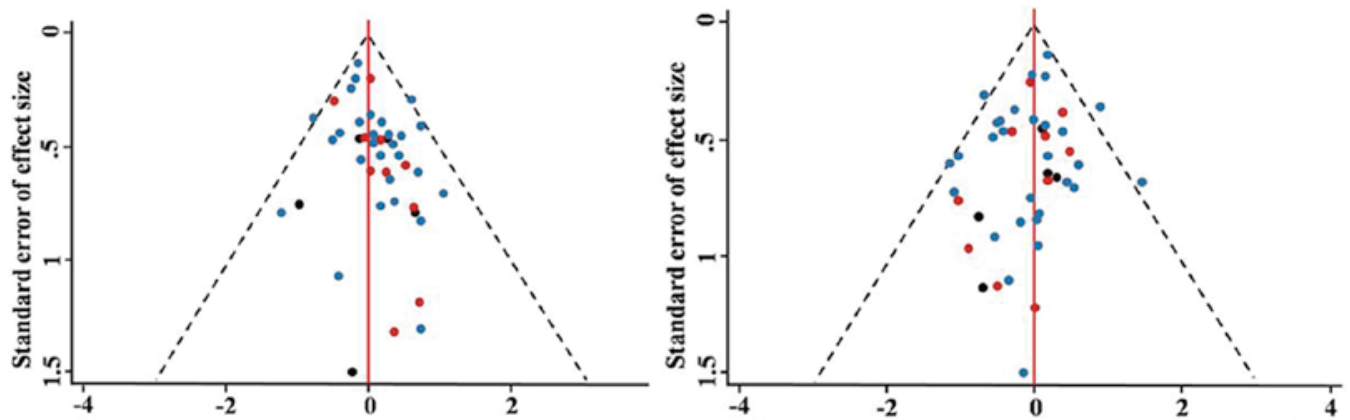

Effect size centered at comparison-specific pooled effect Effect size centered at comparison-specific pooled effect $(y i x Y-\mu x x)$ $(y i X Y-\mu X Y)$

$\mathrm{SD}$

PD
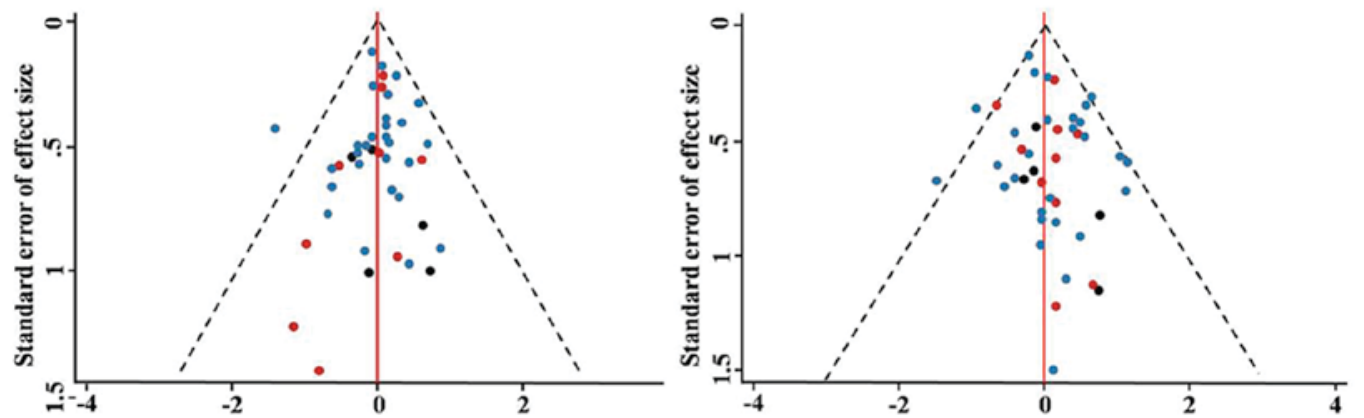

Effect size centered at comparison-specific pooled effect Effect size centered at comparison-specific pooled effect $(y X X Y-\mu x Y)$ 
Rash and diarrhea were the most frequent adverse effects in patients with advanced NSCLC treated with EGFR-TKIs. The network meta-analysis conducted in the current study demonstrated that erlotinib resulted in a higher frequency of nausea and vomiting compared with gefitinib and icotinib. In addition, erlotinib resulted in a higher frequency of rash, nausea and vomiting and fatigue in patients with advanced NSCLC compared with gefitnib and icotinib in the meta-analysis for two congruent drugs and ranking interventions. The adverse events caused by erlotinib treatment can be dose-dependent (10). The approved daily dose of erlotinib $(150 \mathrm{mg})$ is equal to the maximum tolerated dose (10). Therefore, the increase in the toxicity of erlotinib was associated with the dose (10). Furthermore, the adverse effects of erlotinib, icotinib and gefitinib on the liver occur through different mechanisms (62). The different toxicity profiles of these drugs are due to differences in their chemical structure and pharmacokinetics $(63,64)$.

The results of the current study revealed that erlotinib had a longer MST compared with gefitinib and icotinib. Wu et al (46) reported that erlotinib had a higher efficacy compared with gefitinib for the treatment of patient with NSCLC patients without activating mutations of EGFR. The longer MST following erlotinib treatment might be associated with different characteristics of the population such as the mutation of EGFR in the population. Previous studies have revealed that the efficacy of EGFR-TKIs can be associated with the appearance of rash; the more frequent the rash, the more effective the drug was for the treatment of NSCLC $(65,66)$. Therefore, the increased frequency of rash observed following erlotinib treatment may be associated with its longer MST. In the present study, the network meta-analysis also indicated that erlotinib may have a higher SD rate compared with erlotinib and icotinib.

The efficacy of EGFR-TKIs in the treatment of patients with advanced NSCLC may be associated with gender, smoking, ethnicity and tumor pathology (47). However, these clinical factors could not be accounted for in the current analysis due to the limited number of studies evaluated. The present study was also limited by its retrospective nature and the heterogeneity of the treatment regimens.

In the present study, the most common adverse events of EGFR-TKIs in patients with advanced NSCLC were rash and diarrhea. The efficacy of these drugs may be associated with the frequency of rash $(65,66)$; however, the underlying molecular mechanism by which this occurs remains unknown. EGFR-TKI efficacy may also be associated with gender, smoking, ethnicity, tumor pathology, pharmacokinetics and population characteristics $(47,63,64)$.

In conclusion, the results of the present study indicate that gefitinib, erlotinib and icotinib exhibit similar efficacy in the treatment of patients with advanced NSCLC. Erlotinib may increase survival rates compared with gefitinib or icotinib, but more frequently results in side effects. Further clinical trials evaluating the efficacy of erlotinib, icotinib and gefitinib are required.

\section{Acknowledgements}

The present study was supported by Xinjiang Production and Construction Corps (grant nos. 2014BA039 and 2015AG014), and Shihezi University (grant no. GJHZ201602).

\section{References}

1. Lee VW, Schwander B and Lee VH: Effectiveness and cost-effectiveness of erlotinib versus gefitinib in first-line treatment of epidermal growth factor receptor-activating mutation-positive non-small-cell lung cancer patients in Hong Kong. Hong Kong Med J 20: 178-186, 2014.

2. Chen WQ, Zheng RS, Zeng HM, Zou XN, Zhang SW and He J: Report of cancer incidence and mortality in China, 2011. China Cancer 24: 1-10, 2015.

3. Li S, Zhang X, Yan Y, Wang K, Rui D, Pang L and Li F: High cancer burden in elderly chinese, 2005-2011. Int J Environ Res Public Health 12: 12196-12211, 2015.

4. Kim ST, Uhm JE, Lee J, Sun JM, Sohn I, Kim SW, Jung SH, Park YH, Ahn JS, Park K and Ahn MJ: Randomized phase II study of gefitinib versus erlotinib in patients with advanced non-small cell lung cancer who failed previous chemotherapy. Lung Cancer 75: 82-88, 2012.

5. Ellis PM, Coakley N, Feld R, Kuruvilla S and Ung YC: Use of the epidermal growth factor receptor inhibitors gefitinib, erlotinib, afatinib, dacomitinib and icotinib in the treatment of non-small-cell lung cancer: A systematic review. Curr Oncol 22: e183-215, 2015 .

6. Liu J, Sheng Z, Zhang Y and Li G: The Efficacy of epidermal growth factor receptor tyrosine kinase inhibitors for molecularly selected patients with non-small cell lung cancer: A meta-analysis of 30 randomized controlled trials. Targ Oncol 11: 49-58, 2016.

7. Fukuoka M, Yano S, Giaccone G, Tamura T, Nakagawa K, Douillard JY, Nishiwaki Y, Vansteenkiste J, Kudoh S, Rischin D, et al: Multi-institutional randomized phase II trial of gefitinib for previously treated patients with advanced non-small-cell lung cancer. J Clin Oncol 21: 2237-2246, 2003.

8. Thatcher N, Chang A, Parikh P, Rodrigues Pereira J, Ciuleanu T, von Pawel J, Thongprasert S, Tan EH, Pemberton K, Archer V and Carroll K: Gefitinib plus best supportive care in previously treated patients with refractory advanced non-small-cell lung cancer: Results from a randomised, placebo-controlled, multicentre study (iressa survival evaluation in lung cancer). Lancet 366: 1527-1537, 2005.

9. Shepherd FA, Rodrigues Pereira J, Ciuleanu T, Tan EH, Hirsh V, Thongprasert S, Campos D, Maoleekoonpiroj S, Smylie M, Martins R, et al: Erlotinib in previously treated non-small-cell lung cancer. N Engl J Med 353: 123-132, 2005.

10. Togashi Y, Masago K, Fujita S, Hatachi Y, Fukuhara A, Nagai H, Sakamori Y, Kim YH, Mio T and Mishima M: Differences in adverse events between $250 \mathrm{mg}$ daily gefitinib and $150 \mathrm{mg}$ daily erlotinib in Japanese patients with non-small cell lung cancer. Lung Cancer 74: 98-102, 2011.

11. Fan WC, Yu CJ, Tsai CM, Huang MS, Lai CL, Hsia TC, Tien YJ, Huang SF, Wu CH, Chou KT, et al: Different efficacies of erlotinib and gefitinib in taiwanese patients with advanced non-small cell lung cancer: A retrospective multicenter study. J Thorac Oncol 6: 148-155, 2011.

12. Urata Y, Katakami N, Morita S, Kaji R, Yoshioka H, Seto T, Satouchi M, Iwamoto Y, Kanehara M, Fujimoto D, et al: Randomized phase III study comparing gefitinib with erlotinib in patients with previously treated advanced lung adenocarcinoma: WJOG 5108 L. J Clin Oncol 34: 3248-3257, 2016.

13. Xia J, Si RR and Wu YF: Clinical research on icotinib hydrochloride in the treatment of advanced non-small cell lung cancer. J Basic Clin Oncol 28: 210-212, 2015.

14. Shi Y, Zhang L, Liu X, Zhou C, Zhang L, Zhang S, Wang D, Li Q, Qin S, Hu C, et al: Icotinib versus gefitinib in previously treated advanced non-small-cell lung cancer (ICOGEN): A randomised, double-blind phase 3 non-inferiority trial. Lancet Oncol 14: 953-961, 2013.

15. Li YP: Evidence-based Medicine. People's Medical Publishing House, Beijing, 2014.

16. Zeng XT, Bao CP, Cao SY and Liu JY: The quality assessments for randomized controlled trials. Chin J Evid Based Cardiovasc Med 4: 183-184, 2012.

17. Zeng XT, Liu H, Chen $X$ and Leng WD: The quality assessments for observational study. Chin J Evid Based Cardiovasc Med 4: 297-299, 2012.

18. Yuhara H, Steinmaus C, Cohen SE, Corley DA, Tei Y and Buffler PA: Is diabetes mellitus an independent risk factor for colon cancer and rectal cancer? Am J Gastroenterol 106: 1911-1921, 2011 
19. Therasse P, Arbuck SG, Eisenhauer EA, Wanders J, Kaplan RS, Rubinstein L, Verweij J, Van Glabbeke M, van Oosterom AT, Christian MC and Gwyther SG: New guidelines to evaluate the response to treatment in solid tumors. European organization for research and treatment of cancer, national cancer institute of the United States, national cancer institute of Canada. J Nati Cancer Inst 92: 205-216, 2000.

20. Trotti A, Colevas AD, Setser A, Rusch V, Jaques D, Budach V, Langer C, Murphy B, Cumberlin R, Coleman CN and Rubin P: CTCAE v3.0: Development of a comprehensive grading system for the adverse effects of cancer treatment. Semin Radiat Oncol 13: 176-181, 2003.

21. Yi YX, Zhang W, Liu XY, Zhang J, Zhu DJ and Lv QY: Result interpretation of network meta-analysis. Chin J Evidence Based Med 15: 103-109, 2015.

22. Wang HY and Zhang DF: Comparison of the efficacy of gefitinib and erlotinib as a second line treatment for advanced non-small cell lung cancer. J Practical Med 28: 3444-3446, 2012.

23. Song C, Xu LY, Qiao JJ, Li M, Zhao JB and Sun LM: Efficacy and safety of EGFR-TKIs as first-line treatment in 112 elder patients with advanced non-small cell lung cancer. J Dalian Med Univ 37: 282-285, 2015.

24. Wu X, Zhang HY, Lv WZ and Lin Z: Comparation of clinical effects and safety between gefitinib and erlotinib in treatment of patients with NSCLC. Chin J of Misdiagnostics 11: 3534-3536, 2011.

25. Yuan HF: Comparison of clinical effectiveness of gefitinib and erlotinib on non-small cell lung cancer. Sci and Tech of West China 14: 108-109, 2015.

26. Qu WF: Clinical effect of erlotinib on non - small cell lung cancer and its impact on immunoglobulin levels and T-lymphocyte subsets. Practical J of Cardiac Cerebral Pneumal and Vascular Disease 23: 73-75, 2015

27. Wang YX: Comparation of the efficacy and care between gefitinib and erlotinib for the patients with NSCLC. Medical Inform 27: 174-175, 2014

28. Xie Y, Liang J and Su N: Gefitinib versus erlotinib as first-line treatment for patients with advanced EGFR mutation-positive non-small-cell lung cancer. Nan Fang Yi Ke Da Xue Xue Bao 35 446-449, 2015 (In Chinese).

29. Xie YK: The clinical effects and safety of gefitinib for patients with non-small cell lung cancer. Yiayao Qianyan 4: 192-192, 2014

30. Weng XL: Clinical effect and pharmacoeconomics of gefitinib and erlotinib in advanced non-small-cell lung cancer. Chin Med Pharm 5: 100-102, 2015.

31. Zhang J, Liu SQ, Zhang J, Ban LY and Zhou T: Effect and cost-efficacy analysis of the second-line treatment of advanced non-small cell lung cancer. Chin Clin Oncol 17: 908-911, 2012.

32. Zhang CW: The observation of toxicities for targeted therapy in advanced non-small cell lung cancer. Hainan Med J 25: 2273-2274, 2014

33. Chen XP, Hang XS, Gao X, Xu WH, Li C and Zhao J: Adverse drug reaction of gefitinib in therapy for patients with advanced non-small cell lung cancer. Chin J of Hemorheology 19: 579-582, 2009.

34. Zhang YQ, Li YP, Ni J and Liu GL: Clinical effect and pharmacoeconomics of gefitinib and erlotinib in advanced non-small-cell lung cancer. Chin J New Drugs Clin Rem 28: 837-840, 2009.

35. Zhang YJ, Li HB, Li XD, Liu XC and Han JC: Clinical effect and safety of gefitinib and erlotinib second line treatment of lung adenocarcinoma. Chin J Clin Pharmacol 31: 899-901, 2015.

36. Li YY, Li L and Lv EJ: The comparison of efficacy between gefitinib and erlotinib for patients with brain metastases from non-small cell lung cancer. Chin J Clin Res 28: 1308-1311, 2015.

37. Bai H, Xiong LW, Han BH and Jiang LY: Clinical observation of gefitinib and erlotinib for brain metastases of non-small cell lung cancer. Chin Clin Oncol 20: 1028-1031, 2015.

38. Li L: Clinical observation of 70 patients with brain metastases. Dalian Medical Univ, 2013.

39. Zhang JX, Cai D, Li SY, Zhou CZ, Qin YY and Ouyang M: Clinical comparison of erlotinib and gefitinib in non-small cell lung cancer with brain metastases. Chin J Cancer Prevent Treat 22: 285-288, 2015.

40. Ma Y, Huang Y, Zhao H, Liu J, Chen L, Wu H and Zhou N: The cost-effectiveness analysis of gefitinib or erlotinib in the treatment of advanced EGFR mutant non-small cell lung cancer patients. Zhongguo Fei Ai Za Zhi 16: 203-210, 2013 (In Chinese)

41. Shao YY, Shau WY, Lin ZZ, Chen HM, Kuo R, Yang JC and Lai MS: Comparison of gefitinib and erlotinib efficacies as third-line therapy for advanced non-small-cell lung cancer. Eur J Cancer 49: 106-114, 2013
42. Lim SH, Lee JY, Sun JM, Ahn JS, Park K and Ahn MJ: Comparison of clinical outcomes following gefitinib and erlotinib treatment in non-small-cell lung cancer patients harboring an epidermal growth factor receptor mutation in either exon 19 or 21. J Thorac Oncol 9: 506-511, 2014.

43. Yoshida T, Yamada K, Azuma K, Kawahara A, Abe H, Hattori S, Yamashita F, Zaizen Y, Kage M and Hoshino T: Comparison of adverse events and efficacy between gefitinib and erlotinib in patients with non-small-cell lung cancer: A retrospective analysis. Med Oncol 30: 349, 2013.

44. Hong J, Kyung SY, Lee SP, Park JW, Jung SH, Lee JI, Park SH, Sym SJ, Park J, Cho EK, et al: Pemetrexed versus gefitinib versus erlotinib in previously treated patients with non-small cell lung cancer. Korean J Intern Med 25: 294-300, 2010.

45. Emery IF, Battelli C, Auclair PL, Carrier K and Hayes DM: Response to gefitinib and erlotinib in non-small cell lung cancer: A retrospective study. BMC Cancer 9: 333, 2009.

46. Wu WS, Chen YM, Tsai CM, Shih JF, Chiu CH, Chou KT, Lai SL, Wu CH, Luo YH, Huang CY, et al: Erlotinib has better efficacy than gefitinib in adenocarcinoma patients without EGFR-activating mutations, but similar efficacy in patients with EGFR-activating mutations. Exp Ther Med 3: 207-213, 2012.

47. Wu JY, Wu SG, Yang CH, Chang YL, Chang YC, Hsu YC, Shih JY and Yang PC: Comparison of gefitinib and erlotinib in advanced NSCLC and the effect of EGFR mutations. Lung Cancer 72: 205-212, 2011.

48. Kim ST, Lee J, Kim JH, Won YW, Sun JM, Yun J, Park YH, Ahn JS, Park K and Ahn MJ: Comparison of gefitinib versus erlotinib in patients with nonsmall cell lung cancer who failed previous chemotherapy. Cancer 116: 3025-3033, 2010.

49. Chen JH, Luo YZ, Wang W, Zhou WW and Wen XP: Efficacy and toxicity of ecotinib and gefitinib in the treatment for 28 patients with non-small cell lung cancer who have failed previous chemotherapy. Chin J New Drugs 21: 2056-2059, 2012.

50. Chen JH, Luo YZ, Wang W, Zhou WW and Wen XP: A phase III study on icotinib hydrochloride for non-small cell lung cancer. Anti-Tumor Pharmacy 1: 441-443, 2011.

51. Cui HZ, Guan JZ, Liao GQ, Liu PH, Li LL and Shao Y: Efficacy of icotinib and gefitinib in advanced lung adenocarcinoma with EGFR mutation. Acad J Chin Pla Med School 36: 326-328, 341, 2015.

52. Cui HQ, Liu HF and Lv JR: Icotinib in treatment for 49 patients with non small cell lung cancer in epidermal growth factor receptor mutant. Chin J New Drugs Clin Rem 34: 556-559, 2015.

53. Liu AM and Liu HQ: The observation and care of EGFR-TKIs therapy for patients with advanced non-small cell lung cancer. Med Inf 27: 175-176, 2014.

54. Lin WX and Zhang LJ: Clinical observation of icotinib for non-small cell lung cancer. Chin J Prim Med Pharm 21: 106-107, 2014.

55. Xu LJ, Liu H, Li J and Gao Y: Clinical observation of icotinib and gefitinib in first-line therapy for advanced non-small cell lung cancer. J Hunan Normal Univ (Med Sci) 12: 94-97, 2015.

56. Huang Y: Clinical observation of icotinib hydrochloride and erlotinib in the treatment of patients with advanced lung cancer who failed previous chemotherapy. Chin Foreign Med Treat 33: 8-9, 2014

57. Sun Y, Song C, Li M, Zhao JB and Sun LM: The comparison of efficacy between icotinib and erlotinib for patients with advanced non-small cell lung cancer. Shandong Med J 55: 34-36, 2015.

58. Zhang JX, Yu X, Zhang BB, Guan Q, Chen X, Zhang Z, Yang BJ and Zhao MF: Comparison of clinical effects and safety between icotinib and erlotinib in treatment of advanced non-small cell lung cancers. J of Chin Phy 17: 1032-1035, 2015.

59. Hu X, Zhang L, Shi Y, Zhou C, Liu X, Wang D, Song Y, Li Q, Feng J, Qin S, et al: The efficacy and safety of icotinib in patients with advanced non-small cell lung cancer previously treated with chemotherapy: A single-arm, multi-center, prospective study. PLoS One 10: e0142500, 2015.

60. Shao YY, Lin CC and Yang CH: Gefitinib or erlotinib in the treatment of advanced non-small cell lung cancer. Discov Med 9: 538-545, 2010.

61. Niu M, Hu J, Wu S, Xiaoe Z, Xu H, Zhang Y, Zhang J and Yang Y: Structural bioinformatics-based identification of EGFR inhibitor gefitinib as a putative lead compound for BACE. Chem Biol Orug Des 83: 81-88, 2014.

62. Takeda M, Okamoto I, Fukuoka M and Nakagawa K: Successful treatment with erlotinib after gefitinib-related severe hepatotoxicity. J Clin Oncol 28: e273-274, 2010. 
63. Zhang Q and Zhou W: The new choice for non-small cell lung cancer patients - iootinib hydrochloride. J Pharmaceuti Res 32: 121-124, 2013

64. Peters S,Zimmermann S and Adjei AA: Oral epidermal growth factor receptor tyrosine kinase inhibitors for the treatment of non-small cell lung cancer: Comparative pharmacokinetics and drug-drug interactions. Cancer Treat Rev 40: 917-926, 2014.
65. Liu HB, Wu Y, Lv TF, Yao YW, Xiao YY, Yuan DM and Song Y: Skin rash could predict the response to EGFR tyrosine kinase inhibitor and the prognosis for patients with non-small cell lung cancer: A systematic review and meta-analysis. PLoS one 8: e 55128, 2013.

66. Li HR and Sun YF: The severe adverse events and prevention measures for erlotinib. Chin J Pharmacoepidemiol 19: 232-233, 2010. 